\title{
A NOTE ON AN OVERDETERMINED PROBLEM FOR THE CAPACITARY POTENTIAL
}

\author{
CHIARA BIANCHINI AND GIULIO CIRAOLO
}

\begin{abstract}
We consider an overdetermined problem arising in potential theory for the capacitary potential and we prove a radial symmetry result.
\end{abstract}

AMS subject classifications. 35N25, 35B06, 31B15, 35R25, 52A40.

Key words. Overdetermined boundary value problems, electrostatic potential, symmetry, capacity.

\section{INTRODUCTION}

In this note we deal with an overdetermined problem for the electrostatic potential. The electrostatic capacity of a bounded set $\Omega \subset \mathbb{R}^{n}, n \geq 3$, is defined by

$$
\operatorname{Cap}(\Omega)=\inf \left\{\int_{\mathbb{R}^{n}}|D v|^{2} d x: v \in C_{c}^{\infty}\left(\mathbb{R}^{n}\right), v(x) \geq 1 \quad \forall x \in \Omega\right\},
$$

where $C_{c}^{\infty}\left(\mathbb{R}^{n}\right)$ denotes the set of $C^{\infty}$ functions having compact support. It is well-known that it can be equivalently obtained via the asymptotic expansion of the so-called electrostatic potential of $\Omega$ (or capacitary function of $\Omega$ ), i.e.

$$
\operatorname{Cap}(\Omega)=(n-2) \omega_{n} \lim _{|x| \rightarrow \infty} u(x)|x|^{n-2},
$$

where $\omega_{n}$ denotes the surface area of the unit sphere in $\mathbb{R}^{n}$, and $u$ realizes the minimum of problem (1) and hence satisfies

$$
\begin{cases}\Delta u=0 & \text { in } \mathbb{R}^{n} \backslash \bar{\Omega}, \\ u=1 & \text { on } \partial \Omega, \\ \lim _{|x| \rightarrow+\infty} u(x)=0 . & \end{cases}
$$

We mention that the electrostatic potential $u$ represents the potential energy of the electrical field induced by the conductor $\Omega$, normalized so that the voltage difference between $\partial \Omega$ and infinity is one, and hence $\operatorname{Cap}(\Omega)$ represents the total electric charge needed to induce the potential $u$ (see for instance [8]).

A classical question in potential theory is the study of symmetry properties for problem (3). More precisely, one imposes an extra assumption to Problem (3) and studies how such an overdetermination reflects on the domain $\Omega$. In particular, one can ask whether certain geometric properties of the constraint are inherited by the domain $\Omega$. In this respect, a typical problem is the so-called Serrin's exterior problem, where one assumes that

$$
|D u|=c \text { on } \partial \Omega,
$$

where $c$ is a positive constant, and one proves that a solution to (3)-(4) exists if and only if the domain $\Omega$ is a ball. This kind of problem has been successfully solved in [9] by using 
the method of moving planes. Other similar problems and related results can be found in [2, 3, 7, 10, 11, 12].

In this note we discuss two kinds of overdeterminations involving the mean curvature $H_{\partial \Omega}$ of $\partial \Omega$ (that is the average of the principal curvatures of $\partial \Omega$ ). More precisely, we prove the following theorem.

Theorem 1.1. Let $\Omega \subset \mathbb{R}^{n}$ be a bounded domain with boundary of class $C^{2}$ and let $u$ be the solution of (3). If $u$ and $\Omega$ are such that

$$
\int_{\partial \Omega}|D u|^{2}\left[H_{\partial \Omega}-\frac{|D u|}{n-2}\right] d \mathcal{H}^{n-1} \leq 0
$$

or

$$
\int_{\partial \Omega}|D u|^{2}\left[(n-1) H_{\partial \Omega}-\frac{n|D u|}{2(n-2)}\right] d \mathcal{H}^{N-1} \leq \frac{(n-2)^{3}}{2} \omega_{n}\left(\frac{\operatorname{Cap}(\Omega)}{(n-2) \omega_{n}}\right)^{\frac{n-4}{n-2}},
$$

then $\Omega$ is a ball and $u$ is radially symmetric.

We mention that in the case that constraint (5) holds, Theorem 1.1 was already proven in [1]. Indeed, in [1, Theorem 1.1] the authors prove the symmetry result by using a conformal reformulation of the problem and by proving the rotational symmetry via a splitting argument. In this respect, we give a different proof of this theorem.

Our approach is very simple and use a chain of integral identities and a basic inequality for symmetric elementary functions (known as Newton's inequality), as in the spirit of [4, 5, 6]. More precisely, by considering the auxiliary problem for the function

$$
v=u^{-\frac{2}{n-2}}
$$

where $u$ solves (3), we prove that $v$ must be quadratic, and hence the capacitary function $u$ has radial symmetry. This approach is very flexible and it has been extended to more general settings [2, 3].

It is interesting to notice that from the proof of Theorem 1.1 (see Step 1 in Section 3) we immediately obtain the following lower bound for the capacity, for $n=3$ :

$$
\operatorname{Cap}(\Omega) \int_{\partial \Omega}|D u|^{2}\left[(n-1) H_{\partial \Omega}-\frac{n|D u|}{2(n-2)}\right] d \mathcal{H}^{N-1} \geq \frac{(n-2)^{3}}{2} \omega_{n} .
$$

This lower bound is optimal, in the sense that the equality sign is attained when $\Omega$ is a ball.

Acknowledgements. The work has been supported by the FIRB project 2013 "Geometrical and Qualitative aspects of PDE" and the GNAMPA of the Istituto Nazionale di Alta Matematica (INdAM).

\section{PRELIMINARIES}

We use the following notation. Let $A=\left(a_{i j}\right)$ be a $n \times n$ symmetric matrix. We denote by $S_{k}(A), k \in\{1, \ldots, n\}$, the sum of all the principal minors of $A$ of order $k$, so that $S_{1}(A)=$ $\operatorname{tr}(A)$ and $S_{n}(A)=\operatorname{det}(A)$. Denoting by

it holds

$$
S_{i j}^{k}(A)=\frac{\partial}{\partial a_{i j}} S_{k}(A)
$$

$$
S_{k}(A)=\frac{1}{k} S_{i j}^{k}(A) a_{i j}
$$


where here and later the Einstein summation convention is applied. In particular for $k=2$

$$
S_{i j}^{2}(A)=\frac{\partial}{\partial a_{i j}} S_{2}(A)= \begin{cases}-a_{j i} & i \neq j \\ \sum_{k \neq i} a_{k k} & i=j .\end{cases}
$$

Notice that $S_{k}(A)$ are the $k$-th elementary symmetric function of the eigenvalues of $A$; so that

$$
S_{k}(A)=S_{k}\left(\lambda_{1}, \ldots, \lambda_{n}\right)=\sum_{1 \leq i_{1}<\ldots<i_{k} \leq n} \lambda_{i_{1}} \cdot \ldots \cdot \lambda_{i_{n}}
$$

where $\lambda_{i}$ are the eigenvalues of the matrix $A$.

When $A=D^{2} v$ we have that

$$
S_{k}\left(D^{2} v\right)=\frac{1}{k} \operatorname{div}\left(S_{i j}^{k}\left(D^{2} v\right) v_{j}\right),
$$

which follows from the fact that the vector $\left(S_{i 1}^{k}\left(D^{2} v\right), \ldots, S_{i n}^{k}\left(D^{2} v\right)\right)$ is divergence free for $i=1, \ldots, n$, i.e.

$$
\frac{\partial}{\partial x_{j}} S_{i j}^{k}\left(D^{2} v\right)=0, \quad i=1, \ldots, n .
$$

In particular, for $k=2$ we have

$$
S_{2}\left(D^{2} v\right)=\frac{1}{2} S_{i j}^{2}\left(D^{2} v\right) v_{i j}=\frac{1}{2} \operatorname{div}\left(S_{i j}^{2}\left(D^{2} v\right) v_{j}\right),
$$

where

$$
S_{i j}^{2}\left(D^{2} v\right)=\frac{\partial}{\partial v_{i j}} S_{2}\left(D^{2} v\right)= \begin{cases}-v_{j i} & i \neq j \\ \Delta v-v_{i i} & i=j .\end{cases}
$$

Notice that if $L_{t}=\{v>t\}$ is a super level set of $v$, then

$$
|D v|^{2} \Delta v=(n-1) H_{\partial L_{t}}|D v|^{3}+v_{i} v_{i j} v_{j} \quad \text { on } \partial L_{t},
$$

so that if $\partial L_{t}$ is oriented such that $\nu=D v /|D v|$ then

$$
S_{i j}^{2}\left(D^{2} v\right) v_{i} v_{j}=(n-1) H_{\partial L_{t}}|D v|^{3} \quad \text { on } \partial L_{t} .
$$

Two crucial ingredients in the proof of Theorem 1.1 are contained in next lemmas.

Lemma 2.1 (Newton Inequality). Let $A$ be a symmetric matrix in $\mathbb{R}^{n \times n}$; it holds

$$
S_{2}(A) \leq \frac{n-1}{2 n} \operatorname{Tr}(A)^{2} .
$$

Moreover, if $\operatorname{Tr}(A) \neq 0$ and equality holds in (10), then

$$
A=\frac{\operatorname{Tr}(A)}{n} I .
$$

Lemma 2.2. For any smooth function $v$ and $\gamma \in \mathbb{R}$ we have the following identity:

$$
\begin{aligned}
& 2 v^{\gamma} S_{2}\left(D^{2} v\right)= \\
& =\operatorname{div}\left(\frac{\gamma}{2} v^{\gamma-1}|D v|^{2} D v+v^{\gamma} S_{i j}^{2}\left(D^{2} v\right) v_{i}\right)-\frac{3}{2} \gamma v^{\gamma-1}|D v|^{2} \Delta v-\frac{\gamma(\gamma-1)}{2} v^{\gamma-2}|D v|^{4} .
\end{aligned}
$$


Proof. We notice that for $\gamma=0$ (11) is just the definition of $S_{2}$ and then we may assume $\gamma \neq 0$. Identity (11) immediately follows from the following two identities:

$$
\operatorname{div}\left(v^{\gamma} S_{i j}^{2}\left(D^{2} v\right) v_{i}\right)=2 v^{\gamma} S_{2}\left(D^{2} v\right)+\gamma v^{\gamma-1} S_{i j}^{2}\left(D^{2} v\right) v_{i} v_{j},
$$

and

$$
v^{\gamma-1} S_{i j}^{2}\left(D^{2} v\right) v_{i} v_{j}=\frac{3}{2} v^{\gamma-1}|D v|^{2} \Delta v+\frac{\gamma-1}{2} v^{\gamma-2}|D v|^{4}-\frac{1}{2} \operatorname{div}\left(v^{\gamma-1}|D v|^{2} D v\right) .
$$

Identity (12) is readily obtained from $\gamma v^{\gamma-1} v_{i}=\left(v^{\gamma}\right)_{i}$ and

$$
S_{2}\left(D^{2} v\right)=\frac{1}{2} S_{i j}^{2}\left(D^{2} v\right) v_{i j}=\frac{1}{2} \operatorname{div}\left(S_{i j}^{2}\left(D^{2} v\right) v_{i}\right) .
$$

To prove (13) we notice that, since

$$
S_{i j}^{2}\left(D^{2} v\right) v_{i} v_{j}=|D v|^{2} \Delta v-v_{i} v_{j} v_{i j}
$$

we have that

$$
\begin{aligned}
v^{\gamma-1} S_{i j}^{2}\left(D^{2} v\right) v_{i} v_{j} & =v^{\gamma-1}|D v|^{2} \Delta v-v^{\gamma-1} v_{i} v_{j} v_{i j} \\
& =v^{\gamma-1}|D v|^{2} \Delta v+\frac{1}{2}\left[-\operatorname{div}\left(v^{\gamma-1}|D v|^{2} D v\right)+(\gamma-1) v^{\gamma-2}|D v|^{4}+v^{\gamma-1}|D v|^{2} \Delta v\right] \\
& =\frac{3}{2} v^{\gamma-1}|D v|^{2} \Delta v+\frac{\gamma-1}{2} v^{\gamma-2}|D v|^{4}-\frac{1}{2} \operatorname{div}\left(v^{\gamma-1}|D v|^{2} D v\right),
\end{aligned}
$$

which gives (13).

We conclude this section by recalling some well-known properties of the capacitary potential (see [8]) which will be useful for the proof of Theorem 1.1]

$$
\begin{aligned}
& u=\frac{\operatorname{Cap}(\Omega)}{(n-2) \omega_{n}}|x|^{2-n}+o\left(|x|^{2-n}\right), \\
& u_{i}=-\frac{\operatorname{Cap}(\Omega)}{\omega_{n}}|x|^{-n} x_{i}+o\left(|x|^{1-n}\right), \\
& u_{i j}=\frac{\operatorname{Cap}(\Omega)}{\omega_{n}}|x|^{-n}\left(n \frac{x_{i} x_{j}}{|x|^{2}}-\delta_{i j}\right)+o\left(|x|^{-n}\right),
\end{aligned}
$$

as $|x| \rightarrow+\infty$.

\section{Proof of Theorem 1.1}

Step 1. We prove that the reverse inequality holds in (5) and (6). More precisely, we shall prove that if $u$ is a solution of (3), then it satisfies

$$
\int_{\partial \Omega}|D u|^{2}\left(H_{\partial \Omega}-\frac{1}{n-2} \frac{|D u|}{u}\right) d \mathcal{H}^{N-1} \geq 0
$$

and

$$
\int_{\partial \Omega}|D u|^{2}\left((n-1) H_{\partial \Omega}-\frac{n}{2(n-2)} \frac{|D u|}{u}\right) d \mathcal{H}^{N-1} \geq \frac{(n-2)^{3}}{2} \omega_{n}\left(\frac{\operatorname{Cap}(\Omega)}{(n-2) \omega_{n}}\right)^{\frac{n-4}{n-2}} .
$$


The proof of (15) and (16) is based on Lemma2.2 and the Newton Inequality (10) applied to the Hessian matrix of the function $v=u^{-\frac{2}{n-2}}$. Notice that the function $v$ solves

$$
\begin{cases}\Delta v=\frac{n}{2} \frac{|D v|^{2}}{v} & \text { in } \mathbb{R}^{n} \backslash \bar{\Omega}, \\ v=1 & \text { on } \partial \Omega, \\ v \rightarrow \infty & \text { as }|x| \rightarrow+\infty .\end{cases}
$$

Moreover, it follows from (14) that $v$ satisfies

$$
\begin{gathered}
v=\left(\frac{\operatorname{Cap}(\Omega)}{(n-2) \omega_{n}}\right)^{-\frac{2}{n-2}}|x|^{2}+o\left(|x|^{2}\right), \\
v_{i}=2\left(\frac{\operatorname{Cap}(\Omega)}{(n-2) \omega_{n}}\right)^{-\frac{2}{n-2}} x_{i}+o(|x|), \\
v_{i j}=2\left(\frac{\operatorname{Cap}(\Omega)}{(n-2) \omega_{n}}\right)^{-\frac{2}{n-2}} \delta_{i j}+o(1),
\end{gathered}
$$

as $|x| \rightarrow+\infty$.

We are ready to give the proof of (15) and (16). Let $\gamma$ be a fixed parameter to be chosen later and consider (11) applied to the function $v$, solution of (17). From (10) we have that

$$
\begin{aligned}
& v^{\gamma} \frac{n-1}{n}(\Delta v)^{2} \geq \\
& \quad \geq \operatorname{div}\left(v^{\gamma} S_{i j}^{2}(W) v_{i}\right)+\frac{\gamma}{2} \operatorname{div}\left(v^{\gamma-1}|D v|^{2} D v\right)-\frac{3}{2} \gamma v^{\gamma-1}|D v|^{2} \Delta v-\frac{\gamma}{2}(\gamma-1) v^{\gamma-2}|D v|^{4} .
\end{aligned}
$$

Since $v$ satisfies (17), we obtain that

$$
\operatorname{div}\left(v^{\gamma} S_{i j}^{2}\left(D^{2} v\right) v_{i}\right)+\frac{\gamma}{2} \operatorname{div}\left(v^{\gamma-1}|D v|^{2} D v\right) \leq|D v|^{4} v^{\gamma-2}\left(\frac{n}{4}(n-1)-\frac{\gamma}{2}(1-\gamma)+\frac{3}{2} \gamma \frac{n}{2}\right) .
$$

Now, we make our choiche of $\gamma$ so that the right hand side of the above inequality vanishes. This is achieved for $\gamma_{1}=1-n$ and $\gamma_{2}=-n / 2$. Hence, by choosing $\gamma=\gamma_{i}, i=1,2$, we obtain that $v$ satisfies the following inequality in $\mathbb{R}^{n} \backslash \Omega$ :

$$
\operatorname{div}\left(v^{\gamma} S_{i j}^{2}\left(D^{2} v\right) v_{i}\right)+\frac{\gamma}{2} \operatorname{div}\left(v^{\gamma-1}|D v|^{2} D v\right) \leq 0 .
$$

Let $R>0$ be such that $\bar{\Omega} \subset B_{R}$. We integrate the last inequality over $B_{R} \backslash \Omega$ and apply the divergence theorem: from (9) and since $\nu=D v /|D v|$ on $\partial \Omega$ we have that

$$
\begin{aligned}
\int_{\partial \Omega}\left(v^{\gamma}(n-1) H_{\partial \Omega}|D v|^{2}\right. & \left.+\frac{\gamma}{2} v^{\gamma-1}|D v|^{3}\right) d \mathcal{H}^{N-1} \geq \\
& \geq \int_{\partial B_{R}}\left(v^{\gamma} S_{i j}^{2}\left(D^{2} v\right) v_{i} \nu_{B_{R}}^{j}+\frac{\gamma}{2} v^{\gamma-1}|D v|^{2} v_{j} \nu_{B_{R}}^{j}\right) d \mathcal{H}^{N-1},
\end{aligned}
$$

where $\nu_{B_{R}}$ denotes the outer unit normal vector to $B_{R}$. Now we notice that if $\gamma=\gamma_{1}$, then (18) implies that

$$
\lim _{R \rightarrow \infty} \int_{\partial B_{R}} v^{\gamma_{1}} S_{i j}^{2}\left(D^{2} v\right) v_{i} \nu_{B_{R}}^{j}+\frac{\gamma_{1}}{2} v^{\gamma_{1}-1}|D v|^{2} v_{i} \nu_{B_{R}}^{i}=0
$$


while if $\gamma=\gamma_{2}$ then (18) yields that

$$
\lim _{R \rightarrow \infty} \int_{\partial B_{R}} v^{\gamma_{2}} S_{i j}^{2}\left(D^{2} v\right) v_{i} \nu_{B_{R}}^{j}+\frac{\gamma_{2}}{2} v^{\gamma_{2}-1}|D v|^{2} v_{i} \nu_{B_{R}}^{i}=2(n-2) \omega_{n}\left(\frac{\operatorname{Cap}(\Omega)}{(n-2) \omega_{n}}\right)^{\frac{n-4}{n-2}},
$$

since $\partial B_{R}$ is asymptotically a level set of $v$.

By using the fact that $v=1$ on $\Omega$ and coupling (20) and (21), we obtain

$$
\int_{\partial \Omega}|D v|^{2}\left(H_{\partial \Omega}-\frac{1}{2} \frac{|D v|}{v}\right) \geq 0
$$

while from (20) and (22) we find

$$
\int_{\partial \Omega}|D v|^{2}\left((n-1) H_{\partial \Omega}-\frac{n}{4} \frac{|D v|}{v}\right) \geq 2(n-2) \omega_{n}\left(\frac{\operatorname{Cap}(\Omega)}{(n-2) \omega_{n}}\right)^{\frac{n-4}{n-2}} .
$$

By recalling that $v=u^{-\frac{2}{n-2}}$, from the last two inequalities we immediately obtain (15) and (16).

Step 2. From Step 1 we have that the equality sign holds in (5) and (6). This means that the equality sign holds in Newton inequality, which implies that for every $x \in \mathbb{R}^{n} \backslash \Omega$ there exists a constant $\lambda(x)$ such that

$$
D^{2} v(x)=\lambda(x) I d
$$

It is easy to see that $\lambda$ must be constant. Indeed, let $i=1, \ldots, n$ be fixed and chose any $j \neq i$; we have that

which implies that $\lambda$ is constant. Hence,

$$
\partial_{x_{i}} \lambda(x)=\partial_{x_{i}} u_{x_{j} x_{j}}=\partial_{x_{j}} u_{x_{j} x_{i}}=0
$$

$$
D^{2} v=c I d .
$$

From (17) we find that $|D v|$ is constant on every level surface of $v$. In particular, $|D v|$ is constant on $\partial \Omega$ and hence from (8) and (23) we find that $H_{\partial \Omega}$ is constant and by using Alexandrov Theorem we conclude that $\Omega$ is a ball. The proof is complete.

\section{REFERENCES}

[1] V. Agostiniani, L. Mazzieri. Riemannian aspects of potential theory. J. Math. Pures Appl. 104 (2015), 561586.

[2] C. Bianchini, G. Ciraolo. Serrin's overdetermined problems for the anisotropic $p$-Laplacian. Preprint.

[3] C. Bianchini, G. Ciraolo, P. Salani. An overdetermined problem for the anisotropic capacity. Preprint. arXiv:1509.07640.

[4] B. Brandolini, C. Nitsch, P. Salani, C. Trombetti. Serrin-type overdetermined problems: an alternative proof. Arch. Rat. Mech. Anal. 190 (2008), 267-280.

[5] A. Cianchi, P. Salani. Overdetermined anisotropic elliptic problems, Math. Ann. 345 (2009), 859-881.

[6] A. Colesanti, W. Reichel, P. Salani, in preparation.

[7] G. Crasta, I. Fragalà, F. Gazzola. On a long-standing conjecture by Pólya-Szegö and related topics. Z. Angew. Math. Phys. 56 (2005), 763-782.

[8] O.D. Kellogg, Foundations of potential theory, Dover, New York, 1929.

[9] W. Reichel. Radial Symmetry for an Electrostatic, a Capillarity and some Fully Nonlinear Overdetermined Problems on Exterior Domains. Z. Anal. Anwend. 15 (1996), 619-635.

[10] W. Reichel. Radial Symmetry for Elliptic Boundary-Value Problems on Exterior Domains. Arch. Rational Mech. Anal., 137 (1997), 381-394.

[11] P. Salani. A characterization of balls through optimal concavity for potential functions. Proc. Amer. Math. Soc. 143 (2015), 173-183.

[12] Serrin, A symmetry problem in potential theory, Arch. Rational Mech. Anal., 43 (1971), 304-318. 
C. Bianchini, Dip.to di MATEMATICA E INFormatica “U. Dini”, Università degli STUdi di Firenze, Viale Morgagni 67 / A, 50134 FirenZe - ITALY

E-mail address: cbianchini@math . unifi.it

G. Ciraolo, Dip.to di Matematica e Informatica, Università di Palermo, Via ArChirafi 34, 90123, PALERMO - ITALY

E-mail address: giulio.ciraolo@unipa.it 